\title{
Análise de potenciais arranjos produtivos locais de cerâmica e desenvolvimento local: uma aplicação do índice de concentração normalizado
}

\section{RESUMO}

Este artigo aborda os Arranjos Produtivos Locais (APLs) como forma de concentração de empresas e sua relação como o desenvolvimento local. Questiona-se em que medida a formação de APLs contribui para o desenvolvimento local? O aporte teórico apresenta a importância das concentrações de empresas, suas classificações, características particulares e relação com o desenvolvimento local. Tem como objetivo identificar locais com maior potencial para formação de APL de cerâmica, no estado do Pará e sua relevância para o desenvolvimento local. Para identificação dos APLs foi utilizado, o Índice de Concentração Normalizado (ICN), obtido a partir da aplicação da técnica estatística multivariada de componentes principais. O estudo identificou que nove municípios possuem forte potencial para formação de APL cerâmico, representando $74 \%$ do ICN do Estado na atividade, o que pode contribuir com o desenvolvimento local.

Palavras-chave: Arranjo Produtivo Local. Desenvolvimento Local. Índice de Concentração Normalizado.

Keila Regina Mota Negrão keilanegrao@yahoo.com.br Mestranda em Administração. Universidade da Amazônia (UNAMA)

Sérgio Castro Gomes scgomes03@uol.com.br Doutor em Economia (UFV). Universidade da Amazônia (UNAMA)

Eugenia Rosa Cabral

eugeniacabral@unama.br Doutora em Ciências Humanas (UFMG). Universidade da Amazônia (UNAMA) 


\section{INTRODUÇÃO}

Os primeiros estudos sobre concentrações de empresas são do início do século XX com as proposições de Marshall (1982), defendendo a ideia de que a proximidade geográfica entre empresas de um mesmo ramo de atividades poderia gerar vantagens para o grupo. Este assunto vem se desenvolvendo nos últimos anos com estudos sobre redes, aglomerados, clusters, arranjos produtivos locais (APLs) e sistemas locais de produção (SLPs). Essas estruturas organizacionais são percebidas como estratégias para o aumento da competitividade das empresas e como fortes influenciadores de desenvolvimento local.

Localidades com potencial para determinada atividade podem ser beneficiadas pela formação de APLs, como o caso da Itália, com produtores de queijo mozzarella de búfala (LOCKE, 2001); no estado do Rio Grande do Sul: Polo Moveleiro na Serra Gaúcha, o setor Coureiro-calçadista no Vale dos Sinos e os Citricultores Ecológicos no Vale do Caí; em São Paulo: o Polo Moveleiro de Votuporanga; no Piauí: Apicultura em Simplício Mendes; entre outros (MARTINS; VAZ; CALDAS, 2010).

Estudos anteriores apontam que aglomerações produtivas no setor da construção civil (PINHEIRO; SOBREIRA; RAPINI, 2008) e do couro (DALLEMOLE; SANTANA, 2008) possuem considerável influência par a economia paraense. Diante desse cenário, torna-se oportuno aprofundar estudos nessa linha ou identificar outros setores relevantes para o desenvolvimento do Estado.

Rico em jazidas minerais, o Pará possui lugar de destaque na extração de minerais metálicos (ferro, bauxita, zinco, entre outros) e não metálicos, como os utilizados na construção civil (areia, seixo, brita e argila), conforme estudos de Enriquez (2007). Entre os minerais não metálicos, a argila, matéria prima do setor de cerâmica, se destaca. Ressalte-se que a abundância dessa matéria-prima, com qualidade, em território paraense, coloca o Estado em um lugar de destaque na produção de cerâmica, sendo a cerâmica vermelha o segmento mais importante, especialmente na fabricação de tijolos, telhas e objetos ornamentais, decorativos e utilitários.

O crescimento da cerâmica no estado do Pará remete a oportunidades de negócios que podem beneficiar os vários agentes que participam direta ou indiretamente dessa atividade. Pode contribuir significativamente com o desenvolvimento local, mas merece atenção, no longo prazo, por se tratar de uma atividade que demanda uma matéria prima não renovável.

Nesse sentido, questiona-se neste estudo em que medida a formação de APLs de cerâmica contribui para o desenvolvimento local no estado do Pará?

O objetivo deste artigo é, portanto, identificar os locais com maior potencial para formação de APL de cerâmica no estado do Pará e sua relevância para o desenvolvimento local. Especificamente pretende-se identificar os municípios com potencial para indústria da cerâmica no estado do Pará e mapear os principais indicadores desses municípios, analisando de que forma a atividade de cerâmica pode contribuir para o desenvolvimento local.

Para identificação dos APLs foi utilizado, o Índice de Concentração Normalizado (ICN), obtido a partir da aplicação da técnica estatística multivariada de componentes principais. Os dados foram coletados em fontes secundárias do Registro Anual de Informação Social (RAIS) dos anos de 2000, 2006 e 2012 (BRASIL, 2014), produzidos pela Secretaria de Emprego e Salário do Ministério do Trabalho e Emprego (MTE).

Os resultados mostraram que 42,6\% dos municípios do estado do Pará apresentaram dados de emprego formal para a indústria cerâmica, conforme informações da RAIS 2012. Todas as regiões de integração do estado do Pará apresentaram municípios que desempenham atividades no setor de cerâmica, o que demonstra a relevância do setor para o Estado. Cinco das doze regiões de integração possuem metade, ou mais da metade de seus municípios envolvidos nas atividades de cerâmica. Observou-se que nove municípios possuem forte potencial para formação de APL cerâmico, representando $74 \%$ do ICN do Estado na atividade, podendo contribuir com desenvolvimento local.

O artigo está estruturado da seguinte maneira: na seção 2 é apresentado o aporte teórico utilizado para a análise do problema da pesquisa; na seção 3 são apresentados os procedimentos metodológicos adotados na elaboração do estudo; na seção 4 são apresentados os resultados do estudo, analisados à luz do aporte teórico adotado; na seção 5 constam as conclusões. 


\section{CONCENTRAÇÕES DE EMPRESAS E ARRANJOS PRODUTIVOS LOCAIS (APLS)}

Os estudos de Marshall (1982), no início do século XX, sobre as vantagens das concentrações industriais e da eficiência coletiva, marcaram o início de uma série de pesquisas sobre concentrações de empresas. $O$ conceito marshalliano introduz a ideia de que a concentração de indústrias em uma localidade especifica facilita transações entre fornecedores e compradores, gerando ganhos de escala e transformando a economia local (SCHMITT; LOPES; WITTMANN, 2004). A partir dessa perspectiva, novas pesquisas evoluíram originando estudos sobre concentrações identificadas como aglomerados, Clusters, Arranjo Produtivo Local (APL) e Sistema Local de Produção (SLP).

Porter (2009) trabalha os conceitos de aglomerados ou Clusters e APL de forma conjunta, dizendo tratar-se da reunião de empresas inter-relacionadas e instituições correlatas, localizadas geograficamente em determinada área, possuindo elementos comuns e complementares. Ele se refere à concentração de fornecedores especializados, prestadores de serviços, empresas em setores correlatos e outras instituições específicas que competem, mas também cooperam entre si.

Outros autores enxergam essas concentrações com características próprias que as diferenciam umas das outras. Schmitt, Lopes e Wittmann (2004), entendem que aglomerados são modelos mais simples de concentração de empresas especializadas em determinada atividade, localizadas em uma região (uma rua, um bairro, uma cidade ou uma região de um Estado). A sofisticação de um aglomerado pode evoluir para um APL, caracterizado pela inter-relação das empresas, podendo, mais adiante, formar um sistema local de produção, diferenciado pela interdependência entre as empresas e um sistema próprio de governança (Quadro 1).

\begin{tabular}{|c|c|c|}
\hline $\begin{array}{c}\text { Aglomerado } \\
\text { ou Cluster }\end{array}$ & Quadro 1 - Tipos de Concentração de Empresas \\
\hline & Sistema Local de Produção (SLP) \\
\hline
\end{tabular}

Fonte: Schmitt, Lopes e Wittmann (2004, p. 7). 
Na visão de Cassiolato e Lastres (2003), a diferença entre arranjos produtivos locais e sistemas produtivos locais está no nível da inovação, peça fundamental para a competitividade. Os autores apresentam o conceito de sistemas produtivos e inovativos locais, representados por APLs com capacidade de inovação, de competitividade e de promover o desenvolvimento local. Para eles, arranjos produtivos locais são aglomerações territoriais que envolvem agentes econômicos, políticos e sociais, em um conjunto de atividades econômicas específicas.

No entendimento de Cassiolato e Lastres (2003), os APLs envolvem a participação e a interação de empresas produtoras de bens e serviços finais, fornecedoras de insumos e equipamentos, prestadores de consultoria e serviços, comercializadores e clientes, entre outros. Também fazem parte do APL diversas outras instituições públicas e privadas, voltadas para formação e capacitação de recursos humanos (como escolas técnicas e universidades).

Conforme o Grupo RedeSist, APL refere-se a aglomerações territoriais de agentes econômicos, políticos e sociais, que desenvolvem atividades econômicas afins, apresentando vínculos, mesmo que incipientes. Envolvem a participação e interação de empresas e suas formas de representação e associação. Incluem também outras instituições públicas e privadas envolvidas com a formação e capacitação de recursos humanos (escolas técnicas e universidades); pesquisa, desenvolvimento e engenharia; política, promoção e financiamento (CASSIOLATO; LASTRES, 2003).

De acordo com o Serviço Brasileiro de Apoio às Micro e Pequenas Empresas - SEBRAE (2003), um APL é um aglomerado de empresas que estão localizadas em um mesmo território, que apresentam especialização na produção e mantêm vínculos de articulação, interação, cooperação e aprendizagem entre si e com outros atores locais, com o governo ou com associações empresariais, instituições de crédito e instituições de ensino e pesquisa, principalmente universidades.

Na concepção de Castro (2009), APL se refere a um recorte do espaço geográfico (que pode ser um município ou um conjunto de municípios) que apresente sinais de identidade coletiva (sinais sociais, culturais, econômicos, políticos, ambientais, históricos etc.), mantenha ou tenha capacidade de promover a convergência em termos de expectativas de desenvolvimento; estabeleça parcerias e compromissos para manter e especializar os investimentos de cada um dos atores no próprio território; e promova e seja passível de uma integração econômica e social no âmbito local.

Castro (2009) classifica os APLs em três níveis: arranjos incipientes ou embrionários, arranjos em desenvolvimento e arranjos desenvolvidos:

- Arranjos incipientes ou embrionários são aqueles onde há falta de integração entre os agentes, pouca profissionalização da mão de obra, baixos desempenhos, base produtiva simples, falta de cooperação e investimentos e atuam somente no mercado local, o que os torna importantes, pois interferem na arrecadação do município absolvendo mão de obra local.

- Os Arranjos em desenvolvimento são aqueles que possuem gestão com foco setorial, encontram obstáculos ao acesso a serviços especializados, atuam nos mercados locais, estaduais e nacionais, atraem empresas com mão de obra qualificada e incentivam investimentos em competitividade, sendo muito importantes para o desenvolvimento local.

- Os Arranjos desenvolvidos são aqueles cuja articulação resulta da interação, cooperação e aprendizagem possibilitando inovações de produtos e processos e gerando maior competitividade e capacitação social, com vistas à gestão com foco territorial, interação com a comunidade, campo de atuação centrado nos mercados estadual, nacional e internacional, estreito relacionamento comercial com os bancos, base institucional local diversificada e estrutura produtiva ampla e complexa.

A formação de APL ocorre em diferentes setores, em grandes e pequenas economias, em áreas urbanas e rurais e em diversos níveis geográficos (países, estados, metrópole ou cidades). Podem ser formados somente por empresas do mesmo porte ou por empresas de pequeno, médio ou grande porte. Pode variar em tamanho, amplitude e estagio de desenvolvimento (PORTER, 2009).

Para Schiavetto e Alves (2009), um APL é uma estratégia de pequenas organizações para fazerem frente à concorrência. Os APLs de empresas que praticam a cooperação podem reduzir os custos de transação, simplificar as cadeias de suprimento e de distribuição, facilitar financiamentos bancários e cooperar no uso de matérias-primas, equipamentos e mão de obra. 
A formação de aglomerados também impõe novas funções ao governo. Investimentos, sejam públicos ou privados, para a melhoria das condições de aglomerados tendem a gerar benefícios a muitas empresas, o que irá refletir também na sociedade em geral. Assim, os aglomerados assumem importante papel na política econômica. Porter (2009), afirma que políticas macroeconômicas são necessárias, mas não são suficientes para sustentar a competitividade. É fundamental que o governo atue com políticas microeconômicas, que propiciem essa competitividade, como apoio à inovação tecnológica, à pesquisa de base, à formação de mãode-obra, ao fornecimento de infraestrutura moderna, à regulação, à estabilidade jurídica etc.

É fundamental que o governo defina regras e crie incentivos para encorajar a atividade, uma vez que tem importante papel nas políticas fiscais (receitas e despesas), monetárias (taxa de juros) e cambiais (valor de troca da moeda), além das relações com o comércio exterior. Ações nesses âmbitos podem beneficiar significativamente as atividades e formação dos aglomerados. O governo, também, tem o papel de desenvolver e implementar programas de ação econômica de longo prazo que levem a um processo de mudança que mobilize empresas, instituições e os cidadãos, para que possam conhecer a importância das ações governamentais. O progresso econômico muitas vezes é inibido pela falta de conhecimento. O progresso exige o desenvolvimento de mercados internos cada vez mais exigentes (PORTER, 2009).

Para Barquero (2001), desenvolvimento econômico local diz respeito ao processo de crescimento e mudança estrutural que ocorre pela transferência de recursos das atividades tradicionais para as modernas, assim como pelo aproveitamento das economias externas e pela introdução de inovações, determinando a elevação do bem-estar da população de uma cidade ou região. Este conceito está baseado na idéia de que localidades e territórios dispõem de recursos econômicos, humanos, institucionais e culturais, bem como de economias de escala não aproveitadas, que formam seu potencial de desenvolvimento.

Barquero (2002) destaca que o desenvolvimento endógeno propõe-se a atender às necessidades e demandas da população local através da participação ativa da comunidade envolvida. Aponta que mais do que obter ganhos em termos da posição ocupada pelo sistema produtivo local na divisão internacional ou nacional do trabalho, o objetivo é buscar o bem-estar econômico, social e cultural da comunidade local em seu conjunto. Além de influenciar os aspectos produtivos (agrícolas, industriais e de serviços), a estratégia de desenvolvimento procura também atuar sobre as dimensões sociais e culturais que afetam o bem-estar da sociedade.

Para Puga (2003), há uma dificuldade em mapear os APLs, pois não existe um padrão único de surgimento e desenvolvimento desses arranjos. Por esse motivo, os estudos geralmente buscam identificar a relação entre as empresas e instituições no seu entorno, o que requer pesquisas empíricas. Uma alternativa apresentada para mapeamento dos APLs é a identificação da concentração de empresas em determinada localidade, de um setor particular, como o método dos componentes principais, abordado na seção seguinte.

\section{METODOLOGIA DA PESQUISA}

O método utilizado para identificação dos municípios com potencial formação de arranjos produtivos locais de cerâmica no Estado do Pará tem por base a construção do Índice de Concentração Normalizado (ICN), obtido por meio da aplicação da técnica estatística multivariada de componentes principais, tendo como referência Crocco et al. (2003) e Santana (2004). Esse método é utilizado para identificar e mapear os APLs, sendo adotada para indicar os municípios onde existe especialização em dada aglomeração de atividades produtivas (SANTANA, 2004).

Os dados foram coletados em fontes secundárias e utilizou-se o emprego formal como variável econômica para identificação das aglomerações empresariais formais de cerâmica no estado do Pará. As informações utilizadas sobre emprego formal são referentes à categoria Fabricação de Produtos Cerâmicos, provenientes do Registro Anual de Informação Social (RAIS) dos anos de 2000, 2006 e 2012, produzidos pela Secretaria de Emprego e Salário do Ministério do Trabalho e Emprego (MTE).

O emprego formal é uma variável econômica importante que revela as aglomerações empresariais formais nos locais pesquisados (BRITO; ALBUQUERQUE, 2002). Quanto maior a concentração de emprego em uma atividade especifica maior tende a ser a concentração da atividade. Quanto maior a concentração de emprego em um determinado setor, maior a sua força para atrair mais atividades econômicas. 
Para o cálculo do INC, que indicará os municípios com potencial de formação de APL de cerâmica no estado do Pará, foi necessária a identificação de outros três índices, levando em conta três características principais, descritas a seguir.

a) A especificidade da atividade no município: essa característica é determinada pelo Quociente Locacional $(\mathrm{QL})$, que permite determinar o nível de especialização de uma atividade em determinado município. Esse índice possui a seguinte expressão matemática:

$$
\mathrm{QL}=(\mathrm{Ejc} / \mathrm{Ej}) /(\mathrm{EPAc} / \mathrm{EPA})
$$

Onde: Ejc = número de empregos do setor de cerâmica no município j em estudo;

$\mathrm{Ej}=$ número de empregos referentes a todas as atividades ou setores no município j;

$E P A C=$ número de empregos do setor de cerâmica no Estado do Pará;

EPA = número de empregos de todas as atividades ou setores no Estado do Pará.

Se QL > 1, indica que a participação da atividade no município é mais elevada que a participação dessa mesma atividade na média do Estado. Um QL $<1$ significa que não há indicação de especialização na região considerada.

b) O peso da atividade em relação à estrutura empresarial do município. Essa característica é determinada pelo índice de concentração de Hirschman-Herfindahl (IHH), dado por:

$$
\mathrm{IHH}=(\mathrm{Ejc} / \mathrm{EPAc})-(\mathrm{Ej} / \mathrm{EPA})
$$

Onde: os valores de IHH variam de 0 e 1. Para efeito de análise, IHH mais próximo de 0 (zero) indica fraca concentração da atividade analisada; IHH mais próximo de 1 (um) indica alta concentração da atividade nos municípios analisados em relação ao Estado.

c) A importância da atividade no município como um todo. Essa característica e determinada pelo índice de Participação Relativa (PR), como segue:

$$
\mathrm{PR}=(\text { Ejc } / \text { EPAc })
$$

Onde: o PR revela o percentual do setor em todos os municípios do Estado, variando de 0 a 1, sendo que $P R=0$ representa pouca especialização do setor e $P R=1$ representa muita especialização.

A construção do Índice de Concentração Normalizado se dá, portanto, pela combinação dos três índices citados anteriormente e da utilização do método de análise de componentes principais para o cálculo dos pesos $(\theta)$ de cada índice já citado, apresentando como resultado a expressão a seguir:

$$
\operatorname{ICN}(c, j)=\theta(1) \cdot Q L(c, j)+\theta(2) \cdot \operatorname{IHH}(c, j)+\theta(3) \cdot P R(c, j)
$$

Os pesos específicos de cada um dos três indicadores - QL $(c, j), \operatorname{lHH}(c, j)$ e PR(c,j) - foram identificados utilizando os resultados da análise de componentes principais. Essa técnica permite conhecer qual a importância de cada uma das variáveis para a explicação da variância total dos dados. De acordo com Fávero et al (2009), a análise de componentes principais busca uma combinação linear entre as variáveis observadas, de forma a maximizar a variância total explicada. Se considerarmos que as variáveis $\mathrm{X} 1, \mathrm{X} 2, \mathrm{X} 3$ e X4 são altamente correlacionadas, elas serão combinadas de modo a formar um fator que explicará a maior quantidade de variância da amostra. O segundo componente terá a segunda maior quantidade de variância e não será correlacionado com o primeiro, e assim, progressivamente. 
Santana (2004) explica que o cálculo dos pesos deve iniciar pelos resultados dos autovalores ou variâncias relativas de cada componente principal e a variância acumulada, conforme Tabela 1.

Tabela 1 - Autovalores da Matriz de Correlação ou Variância explicada pelos Componentes Principais, a partir da Matriz de Variância-Covariância.

\begin{tabular}{ccc}
\hline Componente Principal & $\begin{array}{c}\text { Variância Explicada ou } \\
\text { autovalores }\end{array}$ & $\begin{array}{c}\text { Proporção da variância } \\
\text { acumulada total }\end{array}$ \\
\hline Componente $\mathrm{CP}_{1}$ & $\lambda_{1}$ & $\lambda_{1}$ \\
Componente $\mathrm{CP}_{2}$ & $\lambda_{2}$ & $\lambda_{1}+\lambda_{2}$ \\
Componente $\mathrm{CP}_{3}$ & $\lambda_{3}$ & $\lambda_{1}+\lambda_{2}+\lambda_{3}$ \\
\hline
\end{tabular}

Fonte: Santana (2004, p. 124).

Em seguida, faz-se necessário calcular a participação relativa de cada um dos indicadores de cada uma das componentes principais, para assim identificar a real importância das variáveis nas componentes. Para isso, é necessário realizar a soma dos valores absolutos dos autovetores com cada componente principal, de acordo com a equação 5:

$$
\Sigma(i, j=1, \ldots, 3) \gamma(\mathrm{i}, \mathrm{j})=\psi \mathrm{i}
$$

A Tabela 2 informa a matriz de coeficientes ou dos autovetores da matriz correlação.

Tabela 2 - Matriz de Coeficientes ou dos Autovetores da Matriz Correlação

\begin{tabular}{cccc}
\hline Indicador de Insumo & Componente $\mathbf{C P}_{\mathbf{1}}$ & Componente $_{\mathbf{C}} \mathbf{P}_{\mathbf{2}}$ & Componente $\mathbf{C P}_{\mathbf{3}}$ \\
\hline $\mathrm{QL}$ & $\gamma_{11}$ & $\gamma_{12}$ & $\gamma_{13}$ \\
$\mathrm{IHH}$ & $\gamma_{21}$ & $\gamma_{22}$ & $\gamma_{23}$ \\
$\mathrm{PR}$ & $\gamma_{31}$ & $\gamma_{32}$ & $\gamma_{33}$ \\
\hline Soma dos coeficientes & $\psi_{1}$ & $\psi_{2}$ & $\psi_{3}$ \\
\hline
\end{tabular}

Fonte: Santana (2004, p. 125).

De posse dos valores da Tabela 2 é possível calcular o valor relativo de cada um dos indicadores em CAD, uma das componentes principais, evidenciando-se assim a real importância das variáveis nas componentes. Para isso, é necessário dividir o valor absoluto de cada auto vetor $\gamma(\mathrm{i}, \mathrm{j})$ pela soma $\psi \mathrm{i}$, associada a cada componente, originando a matriz de autovetores recalculados, conforme Tabela 3:

\begin{tabular}{cccc}
\multicolumn{4}{c}{ Tabela 3 - Matriz de Participação Relativa dos Indicadores em cada Componente Principal } \\
\hline Indicador de Insumo & Componente $\mathbf{C P}_{\mathbf{1}}$ & Componente $\mathbf{C P}_{\mathbf{2}}$ & Componente $\mathbf{C P}_{\mathbf{3}}$ \\
\hline $\mathrm{QL}$ & $\phi_{11}=\left(\left|\gamma_{11}\right| / \psi_{1}\right)$ & $\phi_{12}=\left(\left|\gamma_{12}\right| / \psi_{2}\right)$ & $\phi_{13}=\left(\left|\gamma_{13}\right| / \psi_{3}\right)$ \\
$\mathrm{IHH}$ & $\phi_{21}=\left(\left|\gamma_{21}\right| / \psi_{1}\right)$ & $\phi_{12}=\left(\left|\gamma_{22}\right| / \psi_{2}\right)$ & $\phi_{23}=\left(\left|\gamma_{23}\right| / \psi_{3}\right)$ \\
$\mathrm{PR}$ & $\phi_{31}=\left(\left|\gamma_{31}\right| / \psi_{1}\right)$ & $\phi_{12}=\left(\left|\gamma_{32}\right| / \psi_{2}\right)$ & $\phi_{33}=\left(\left|\gamma_{33}\right| / \psi_{3}\right)$ \\
\hline \multicolumn{4}{c}{ Fonte: Santana (2004, p. 125). }
\end{tabular}

De posse dos $\phi c j$, que representam o peso de cada variável e dos autovalores $\lambda c$, o peso que deve ser atribuído a cada índice específico é dado pela combinação linear dos produtos dos coeficientes pelos correspondentes autovalores, relativos a cada componente principal, conforme equações a seguir: 


$$
\begin{aligned}
& \Theta \mathrm{i}=\Sigma\left(\mathrm{i}, \mathrm{j}={ }_{1}, \ldots,{ }_{3}\right) \phi \mathrm{ij} \lambda \mathrm{i} \\
& \Sigma\left(\mathrm{i}, \mathrm{j}={ }_{1}, \ldots, 3\right) \phi \mathrm{i}=1
\end{aligned}
$$

Os resultados encontrados serão: $\Theta_{1}$ como peso atribuído para o Quociente Locacional, QL; $\Theta_{2}$ como peso atribuído para o índice de concentração de Hirschman-Herfindahl, $\mathrm{IHH} ; \Theta_{3}$ como peso atribuído para a Participação Relativa, PR.

Considerando que a soma dos pesos é igual a 1, a combinação linear dos indicadores na forma padronizada contribui para gerar o índice de concentração normalizado (ICN), onde os coeficientes são os próprios pesos calculados pelo método das componentes principais. Portanto, os pesos específicos para cada índice consideram a participação destes na explicação do potencial de formação das aglomerações produtivas que os municípios estudados apresentam, setorialmente.

Para o estudo em questão, foram utilizados os softwares Microsoft Excel, versão 2007, para organização dos dados e Statistical Package for the Social Sciences - SPSS, versão 20.0, para aplicação da técnica de componentes principais.

\section{ANÁLISE DOS RESULTADOS}

O estado do Pará é formado por 143 municípios alocados em 12 regiões de integração. Do total de municípios paraenses, 61 apresentaram dados de emprego formal para a indústria cerâmica, conforme informações da RAIS 2012, o que corresponde a 42,6\% dos municípios do Estado, conforme Tabela 4.

Tabela 4 - Municípios Paraenses, por Região de Integração, com Atividades na Indústria Cerâmica.

\begin{tabular}{cccc}
\hline $\begin{array}{c}\text { Região de } \\
\text { Integração }\end{array}$ & $\begin{array}{c}\text { Total de } \\
\text { Municípios }\end{array}$ & $\begin{array}{c}\text { Municípios com } \\
\text { Atividade de Cerâmica }\end{array}$ & $\begin{array}{c}\text { \% Atividade } \\
\text { Cerâmica }\end{array}$ \\
\hline Araguaia & 15 & 11 & 73,33 \\
Baixo Amazonas & 12 & 7 & 58,33 \\
Carajás & 12 & 8 & 66,67 \\
Guamá & 18 & 6 & 33,33 \\
Lago de Tucuruí & 7 & 7 & 100,00 \\
Marajó & 16 & 1 & 6,25 \\
Metropolitana & 5 & 2 & 40,00 \\
Rio Caetés & 15 & 3 & 20,00 \\
Rio Capim & 16 & 8 & 50,00 \\
Tapajós & 6 & 2 & 33,33 \\
Tocantins & 11 & 2 & 18,18 \\
Xingu & 10 & 4 & 40,00 \\
\hline Total & 143 & 61 & 42,66 \\
\hline
\end{tabular}

Fonte: Elaborado pelos autores a partir dos dados da RAIS, 2012.

Todas as regiões de integração do estado do Pará apresentaram municípios que desempenham atividades no setor de cerâmica. Isso demonstra a relevância do setor para o Estado. Do total das doze regiões de integração cinco possuem metade, ou mais da metade de seus municípios envolvidos nas atividades de cerâmica (Araguaia, Baixo Amazonas, Carajás, Lago do Tucuruí e Rio Capim), com destaque para a região do Lago de Tucuruí, onde se constatou que $100 \%$ dos municípios que formam a região possuem atividades de cerâmica.

O Índice de Concentração Normalizado foi calculado para cada um dos 61 municípios da amostra, no entanto, para identificação dos potenciais municípios para formação de arranjo produtivo local, adotouse o critério de excluir os municípios cujos ICN's estão abaixo da média do setor da cerâmica no estado do 
Pará. Como resultado final nove municípios foram identificados com forte potencial para formação de APL da indústria cerâmica, representando $74 \%$ do ICN total do estado do Pará.

Esses municípios estão relacionados na Tabela 5.

Tabela 5 - Índice de Concentração Normalizado dos Municípios do Estado do Pará, com potencial para formação de APL da Cerâmica, por Região de Integração.

\begin{tabular}{cccccc}
\hline Região de Integração & & QL $(\mathbf{c}, \mathbf{j})$ & IHH $(\mathbf{c}, \mathbf{j})$ & PR (c,j) & ICN \\
\hline \multirow{2}{*}{ Guamá } & São Miguel do Guamá & 66,51 & 0,31 & 0,32 & 41,19 \\
& Inhangapi & 60,27 & 0,04 & 0,04 & 36,87 \\
& Santa Isabel do Para & 6,03 & 0,04 & 0,05 & 3,76 \\
& Eldorado dos & 45,21 & 0,03 & 0,03 & 27,66 \\
Carajás & Carajás & & & & \\
& Palestina do Para & 7,59 & 0,00 & 0,00 & 4,64 \\
Lago de Tucuruí & Nova Ipixuna & 31,70 & 0,02 & 0,03 & 19,40 \\
Rio Capim & Irituia & 14,07 & 0,02 & 0,02 & 8,62 \\
Tocantins & Acara & 8,93 & 0,03 & 0,03 & 5,51 \\
Xingu & Brasil Novo & 7,25 & 0,00 & 0,01 & 4,44 \\
\hline
\end{tabular}

Fonte: Elaborado pelos autores (2015).

O índice de especificidade da atividade representado pelo Quociente Locacional (QL), apresentou para o estado do Pará, a média de 5,47 considerando o total de municípios da amostra. Dos 61 municípios analisados 42 (69\%) apresentaram valor de QL> 1, indicando que a participação da atividade no município é mais elevada que a participação dessa mesma atividade na média do Estado. Os municípios destacados na Tabela 5 como potenciais para formação de APLs da cerâmica apresentaram elevados índices de especialidade no setor de cerâmica muito acima da média auferida para o estado.

O índice de concentração de Hirschman-Herfindahl $(\mathrm{IHH})$, que representa o peso da atividade em relação à estrutura empresarial do município, apresentou para o Estado do Pará, a média de 0,01 representando pouca concentração da atividade nos municípios estudados. Dos 61 municípios analisados 17 (28\%) apresentaram valor de $\mathrm{IHH}$ diferentes de 0 (zero), mostrando que a concentração da atividade nos municípios em relação ao estado ainda é tímida. Somente o município de São Miguel do Guamá apresentou IHH muito diferenciado em relação aos demais municípios, no valor de 0,31 (Tabela 5) indicando alta concentração da atividade cerâmica no município.

O índice de Participação Relativa (PR), que revela a importância da atividade no município como um todo, apresentou para o Estado do Pará, a média de 0,02 considerando o total de municípios da amostra, o que representa pouca importância da atividade. Dos 61 municípios analisados 33 apresentaram valor de $P R \neq 0$, ou seja, 54\% dos municípios da amostra possuem alguma importância no setor de cerâmica. Entre os municípios destacados na Tabela 5 como potenciais para formação de APLs da cerâmica, o município de São Miguel do Guamá foi quem apresentou maior índice, $\mathrm{PR}=0,32$, considerado baixo, revelando pouca importância, mas, se comparado aos demais municípios da amostra, este possui destaque considerável.

Quanto ao Índice de Concentração Normalizado (ICN), que indica o potencial de formação de APLs, percebeu-se que a região de integração Guamá foi a que apresentou maior número de municípios com potencial de APL da cerâmica. Essa região, também concentrou os dois municípios com maior ICN: São Miguel do Guamá (ICN = 14,19) e Inhangapi $(I C N=36,87)$.

De acordo com o modelo adotado no estudo, a Região de Integração de Carajás aparece com dois municípios com forte potencial para formação de APL, quais sejam: Eldorado dos Carajás $(I C N=27,66)$ e Palestina do Pará $(4,64)$. As demais regiões de integração com municípios potenciais para formação de APL foram: Lago do Tucuruí, Rio Capim, Tocantins e Xingu, cada uma com um município com ICN acima da média do Estado, na indústria cerâmica. 
Destaca-se que apesar de se ter identificado, inicialmente, a atividade de cerâmica em 100\% dos municípios que compõe a Região de Integração do Lago do Tucuruí, somente o município de Nova Ipixuna, com ICN de 19,40 (Tabela 5) apresentou índice acima da média do Estado, ficando em quarto lugar no ranking dos municípios com maior potencial de formação de APL cerâmico no estado do Pará (Figura 1).

A Figura 1 apresenta, em ordem decrescente, os municípios paraenses com maior potencial para formação de APL da indústria cerâmica, de acordo com o Índice de Concentração Normalizado.

Figura 1 - ICN dos Municípios do Pará com Potencial para Formação de APL da Indústria Cerâmica.

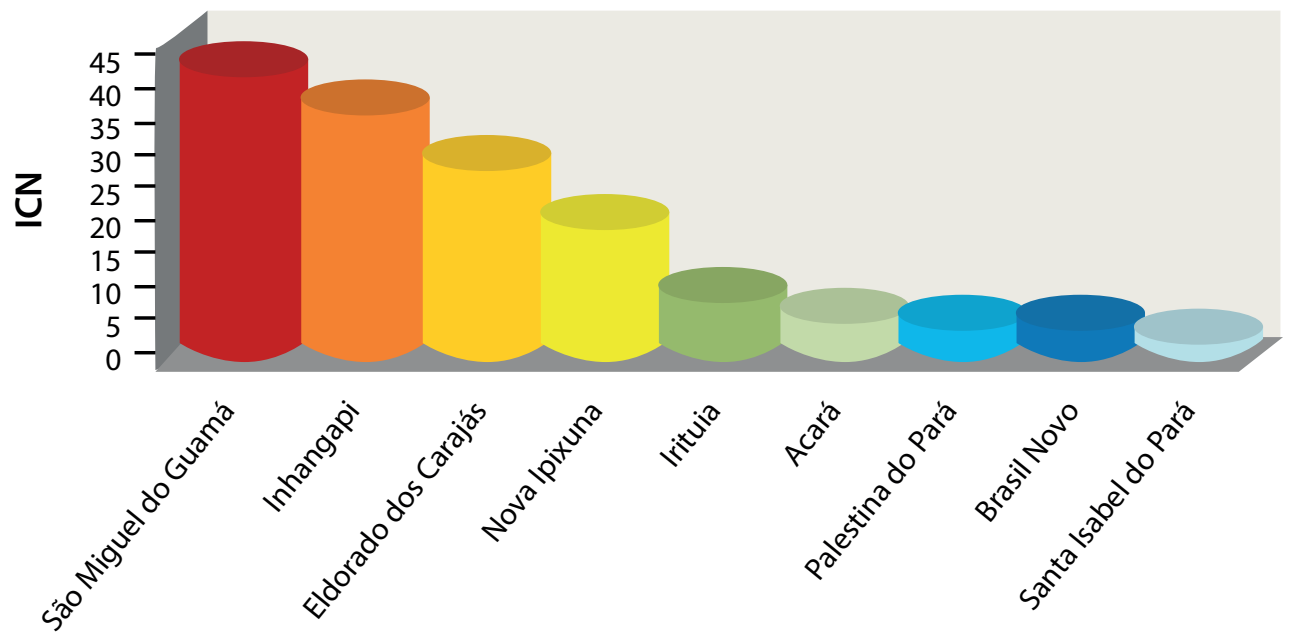

MUNICÍPIOS

Fonte: Elaboração dos autores (2015)

A partir da identificação do ICN dos municípios com potencial para formação de APL, investigou-se o ICN desses municípios para os anos de 2006 e 2000, a fim de verificar o histórico da concentração de cerâmica nesses. Os resultados estão representados na Tabela 6.

Tabela 6 - Série histórica dos Índices de Concentração Normalizado dos Municípios do Pará, com potencial para formação de APL da Cerâmica, segundo a Região de Integração.

\begin{tabular}{ccccc}
\hline Região de Integração & Município-Pará & ICN 2000 & ICN 2006 & ICN 2012 \\
\hline Guamá & São Miguel do Guamá & 60,423 & 56,47 & 41,18 \\
Guamá & Inhangapi & 50,071 & 36,89 & 36,86 \\
Carajás & Eldorado dos Carajás & - & $1,33^{*}$ & 27,66 \\
Lago de Tucuruí & Nova Ipixuna & - & $0,23^{*}$ & 19,39 \\
Rio Capim & Irituia & 22,939 & 12,03 & 8,62 \\
Tocantins & Acará & - & $1,14^{*}$ & 5,51 \\
Carajás & Palestina do Pará & - & 7,65 & 4,63 \\
Xingu & Brasil Novo & - & $2,78^{*}$ & 4,43 \\
Guamá & Santa Isabel do Pará & $5,190^{*}$ & 4,09 & 3,75 \\
\hline
\end{tabular}

Fonte: Elaborado pelos autores a partir dos dados da RAIS 2000, 2006 e 2012.

O estado do Pará apresentou médias para o ICN nos valores de 7,31; 3,67 e 3,36, para os anos de 2000, 2006 e 2012, respectivamente. Os valores identificados na Tabela 6 com o símbolo * representam valores de ICN abaixo da média do Estado para o ano em estudo. 
A projeção história revelou que os municípios de São Miguel do Guamá e Inhangapi, vêm apresentando redução do ICN no decorrer dos anos, provavelmente em decorrência do crescimento da atividade em outros municípios. No entanto, esses municípios se mantêm com os maiores valores para o ICN, indicando predominância da atividade cerâmica forte, durante a última década. Merece destaque, também, os municípios de Eldorado dos Carajás e Nova Ipixuna quanto ao crescimento da atividade de cerâmica em seus territórios. No ano de 2006, na base de dados da RAIS, não foram identificados empregos formais para a atividade "Fabricação de Produtos Cerâmicos", esses dados foram encontrados na base de dados do ano de 2006, em pequena participação, e seis anos depois, os referidos municípios alcançam ICN elevado, o que os coloca entre os quatro no ranking de municípios com maiores potenciais para formação de APL cerâmico (Tabela 6).

De posse desses resultados, foram investigados indicadores socioeconômicos (Tabela 7) e informações sobre a atividade de cerâmica nos municípios de São Miguel do Guamá e Eldorado dos Carajás, a fim de verificar as características de dois dos municípios com maior potencial para formação de APL da Cerâmica. A opção pelos municípios se deu, por estarem localizados em diferentes regiões de integração em que houve a identificação de mais de um município em potencial para formação de APL da indústria cerâmica.

Tabela 7 - Indicadores Socioeconômicos dos municípios Paraenses de São Miguel do Guamá e Eldorado dos Carajás.

\begin{tabular}{ccc}
\hline Informações & \multicolumn{2}{c}{ Municípios } \\
\cline { 2 - 3 } Ano de fundação & São Miguel do Guamá & Eldorado dos Carajás \\
Região de Integração & 1891 & 1991 \\
População (Hab.) - ano 2013 & Guamá & 32.420 \\
Área (km²) - ano 2013 & 54.417 & $2.956,70$ \\
Razão de sexo - ano 2010 & $1.110,00$ & 10,96 \\
Taxa de Urbanização - ano 2010 & 49,02 & 1,12 \\
Habitantes / Unidades Domiciliares - ano 2010 & 1,02 & 52,16 \\
População Economicamente Ativa - ano 2010 & 61,83 & 3,95 \\
População Ocupada - ano 2010 & 4,08 & 12.434 \\
IDH - ano 2010 & 21.306 & 11.476 \\
PIB - ano 2010 & 20.166 & 0,56 \\
\hline
\end{tabular}

Fonte: IDESP (2014).

Com base na Tabela 7, é possível comparar os indicadores de dois dos nove municípios destacados com alto potencial para a formação de APL ceramista no Pará. As estatísticas apontam dados relevantes, como a permanência de um polo cerâmico por longo tempo (São Miguel do Guamá) e o crescimento acelerado de um jovem polo (Eldorado dos Carajás)'1.

De acordo com Cardoso (2013), no município de Eldorado do Carajás existem treze empresas que fabricam e comercializam produtos de cerâmica. Elas atuam principalmente no ramo de cerâmica vermelha, representadas por tijolos, blocos, telhas, elementos vazados, lajes, tubos cerâmicos e argilas expandidas, conforme classificação da Associação Brasileira de Cerâmica. As empresas utilizam a argila natural como matéria-prima e a maioria adota o método tradicional de produção, com forno de chama reversível. Apenas uma das empresas utiliza o forno tipo túnel. Esses dados comprovam pouco, ou nenhum indício de inovação no processo produtivo que possa gerar ganhos de competitividade e levar ao desenvolvimento local. A possível resistência ou falta de cooperação das empresas para articulação de processos produtivos inovadores tornam distante a ideia de formação de um sistema produtivo local, teorizada por Cassiolato e Lastres (2003).

A fabricação dos produtos de cerâmica é realizada em duas etapas: a extração da matéria-prima, no caso, a argila; e o processo de transformação em produto final. "Independentemente de essas fases serem ou não desempenhadas pela mesma empresa, elas estão intimamente interligadas e interfere no desempenho de todo o processo produtivo" (CARDOSO, 2013, p. 15). Esta informação indica os primeiros e possíveis processos de cooperação necessários para a formação de um APL. Um APL pode reduzir os custos de transação, simplificar

1 As informações sobre a indústria de cerâmica em Eldorado do Carajás foram retiradas do Relatório de Gestão e Pesquisa de Mercado do Polo Cerâmico de Eldorado dos Carajás (CARDOSO, 2013). 
as cadeias produtivas, facilitar financiamentos, além da cooperação no uso de matérias-primas, equipamentos e mão de obra dentre outras vantagens, gerando os primeiros sinais de identidade coletiva (SCHIAVETTO; ALVES, 2009; CASTRO, 2009).

Em todo processo de produção da cerâmica predominam as experiências acumuladas pelos ceramistas locais. Essa informação remete à importância da especialização do trabalho e do compartilhamento de informações para alcance de melhores resultados. Para isso, pode-se investir em um estreitamento maior com outros agentes que possam contribuir com treinamentos para especialização do trabalho, pesquisas para melhoria de processos, entre outras ações que envolvem a participação de universidades, governo, associações cooperativas e instituições de crédito, valiosos parceiros no processo de articulação de APLs. (SEBRAE, 2003).

No polo ceramista de Eldorado dos Carajás, os principais produtos comercializados são: tijolos, 91,5\%; telhas, $8 \%$; e lajotas, $0,5 \%$. No processo produtivo, foi identificado que $100 \%$ das empresas adotam a lenha como propulsora de energia e apenas $23 \%$ delas empregam o glicerol, um óleo combustível, considerado uma fonte de energia renovável e ambientalmente correta, como uma alternativa de fonte de energia. Demonstrando certa preocupação com o meio ambiente, uma dimensão importantíssima na promoção do desenvolvimento local.

Quanto às informações do polo ceramista de São Miguel do Pará, serão apresentados dados oriundos da pesquisa empírica e dados secundários, com destaque para os achados de Cordovil e Nahum (2011).

Segundo dados do Sindicato das Indústrias Cerâmicas de São Miguel do Guamá e Região (SINDICER) existem 42 indústrias de cerâmica vermelha ativas nos municípios de Ipixuna do Pará e São Miguel do Guamá. Conforme Cordovil e Nahum (2011), na década de 1980, quando as indústrias de cerâmica começaram a ser instaladas no município de São Miguel do Guamá, ocorreram migrações de trabalhadores de outros municípios do Pará e até de outros Estados, o que gerou o aumento da produtividade, dos fluxos e da pressão sobre o recurso mineral argila. Tal fato ocorreu porque a atividade industrial ceramista assumiu o papel de principal empreendimento econômico que aportou no lugar.

Em São Miguel do Guamá, as atividades que envolvem a cerâmica vermelha predominam, também. Apesar de o município desenvolver atividades relacionadas à indústria de café e madeira, a cerâmica é a atividade considerada de maior importância, pela elevada oferta de emprego e geração de renda (CORDOVIL; NAHUM, 2011). O setor de cerâmica de São Miguel do Guamá responde pela maioria dos postos de trabalho com carteira assinada no município, fator determinante para o desenvolvimento local. O forte potencial para geração de empregos e o elevado índice de postos de trabalho são características fortes para a formação de APL. Além disso, a oferta de emprego pode refletir aumento do negócio, incentivando empreendedores informais a se organizem como pessoa jurídica.

A maior parte da produção da indústria de cerâmica de São Miguel do Guamá é destinada ao mercado paraense e uma pequena parte ao estado do Maranhão. Essa característica específica de distribuição da produção caracteriza o município de São Miguel do Guamá como um Arranjo em desenvolvimento, conforme uma das três classificações de Castro (2009) para APL. Os Arranjos em desenvolvimento atuam nos mercados locais, estaduais e nacionais, atraindo empresas com mão de obra qualificada e incentivando investimentos em competitividade, sendo muito importantes para o desenvolvimento local.

Quanto à produção local, esta iniciou com processos artesanais e foi se modernizando no decorrer dos tempos. A produção, antes manual, tornou-se mecanizada, desde a extração de argila até a fabricação dos moldes de cerâmicas vermelhas. $\mathrm{O}$ tempo de fabricação das peças foi reduzindo à medida que aumentou a necessidade de se obter padrões de produção para atender ao mercado consumidor, atrelados à necessidade de redução de custos. A introdução de máquinas elétricas no processo produtivo aumentou de forma significativa a quantidade de peças produzidas.

Vê-se que a inovação tecnológica, em vários aspectos, gerou benefícios imensuráveis na produção de cerâmica. A esse respeito destaca-se o uso de desintegrador, máquina que desintegra pedras e partículas sólidas, evita danos nas mercadorias e nos equipamentos contribuindo para a diminuição de perdas na etapa de extrusão. A etapa da extrusão é quando a matéria-prima passa pela maromba, que após receber a argila laminada a comprime por uma boquilha, produzindo a forma final do produto que se quer obter (CORDOVIL; NAHUM, 2011). Essa evolução aponta claramente as vantagens competitivas oriundas da inovação, tão necessária nos APLs.

Até o final da década de 1990, os empresários locais atuavam de forma isolada. Em 1998 foi criado o Sindicato da Indústria e da Construção Civil e do Mobiliário, contando com a participação de todos os empresários do setor de cerâmica. Esse processo representa um marco significativo nas atividades de cerâmica do município e uma etapa fundamental para o aprimoramento do APL, pois as relações com instituições públicas e privadas garantem melhores desempenhos aos APLs (PORTER, 2009; CASSIOLATO; LASTRES, 2003).

O município de São Miguel do Guamá apresenta-se em um estágio bastante avançado nas características que determinam um APL. Apesar de sua produção estar perdendo mercado, devido à expansão da atividade 
para outros municípios, pelas experiências que guarda este município pode se desenvolver ainda mais e também servir de exemplo para ações em novas regiões, como o caso da Região de Integração de Carajás, que vem crescendo rapidamente, com destaque para o município de Eldorado de Carajás como polo ceramista de alto potencial para formação de APL.

\section{CONCLUSÃO}

Este artigo se propôs a identificar os locais com maior potencial para formação de arranjo produtivo local de cerâmica no estado do Pará, destacando a relevância dessa atividade e da formação de APLs para o desenvolvimento local.

Os resultados do estudo indicaram a existência de nove municípios com forte potencial para formação de APL da indústria cerâmica no estado do Pará. Verifica-se uma desconcentração da atividade na Região de Integração Guamá, onde tradicionalmente se desenvolvia essa atividade, e uma concentração na Região de Integração Carajás, o que mostra uma expansão da atividade e a existência de um mercado promissor para investimentos, podendo contribuir com o desenvolvimento local, pelas vantagens que a formação de um APL pode proporcionar aos agentes envolvidos e à região como um todo.

Vê-se que a dinâmica produtiva da indústria de cerâmica, em nível local, nos dois municípios identificados com potenciais APLs (São Miguel do Guamá e Eldorado dos Carajás), impacta no desenvolvimento da economia do município, avaliado com base nos dados do PIB municipal. O PIB de Eldorado dos Carajás passou de R\$26.336, em 2000 (74ª posição no ranking estadual), para R\$199.893, em 2010 (51 a posição no ranking estadual); o PIB de São Miguel do Guamá passou de R\$54.805, em 2000 (43a posição no ranking estadual), para R\$201.904, em 2010 (50ª posição no ranking estadual).

O aprofundamento das análises sobre a dinâmica dos potenciais APLs necessita de levantamento de dados primários para cada caso específico, para identificação das atividades, das tecnologias utilizadas, das relações e, entre outros fatores, dos agentes envolvidos no processo de cerâmica, sejam fornecedores, clientes, universidades, sindicatos, associações, empresários, enfim, todos os personagens importantes que caracterizam a formação de um APL.

Os aportes teóricos apontam a relação direta entre APL e desenvolvimento regional e local, todavia, há necessidade de criação de indicadores que possam medir esse desenvolvimento, o que demanda melhor investigação das condições locais, com base em microdados.

A atividade de cerâmica é uma entre as diversas atividades desenvolvidas no Pará e a utilização do método para identificar outras atividades potenciais para o Estado poderia contribuir para abertura e incentivo de novos negócios que possam impulsionar o desenvolvimento local.

Diversos estudos já foram realizados com o objetivo de subsidiar os debates sobre APLs no estado do Pará. Entre esses, alguns destacam que as organizações com potencial para compor os arranjos não conseguem cooperar entre si e, portanto, não são capazes de se fortalecer. Esse aspecto merece mais estudo para identificação das dificuldades de cooperação nesses arranjos para possíveis proposições de melhoria.

Estudos sobre concentrações de empresas e desenvolvimento local são relevantes para a administração empresarial, à gestão de políticas públicas e à sociedade em geral. 


\title{
ANALYSIS OF LOCAL PRODUCTION ARRANGEMENTS OF CERAMIC PRODUCTS AND LOCAL DEVELOPMENT: AN APPLICATION OF THE STANDARDIZED CONCENTRATION INDEX
}

\begin{abstract}
This article discusses the concentration of businesses and local development. It is questionable to what extent the formation of local clusters contributes to local development. The theoretical framework presents the importance of concentrations between undertakings, their classifications, particular characteristics and relationship with local development. It aims to identify sites with the greatest potential for formation of local pottery clusters in the state of Pará and their relevance to local development. For the identification of clusters we used, the Standardized Concentration Index, obtained from the application of multivariate statistical technique of the main components. It was observed that nine municipalities have strong potential for the formation of ceramic LPA (Local Productive Arrangements), representing $74 \%$ of the activity in the SCI (Standardized Concentration Index) in the state and they may contribute to local development.
\end{abstract}

Keywords: Local Productive Arrangement. Local Development. Standardized Concentration Index.

\section{REFERÊNCIAS}

BARQUERO, Antônio Vásquez. Desenvolvimento endógeno em tempos de globalização. Porto Alegre: Fundação de Economia e Estatística, 2002.

BRASIL. Ministério do Trabalho e Emprego. Relação anual de informações sociais, referentes a 2000, 2006 e 2012. Disponível em: <http://portal.mte.gov.br/rais/>. Acesso em: 21 abr. 2014.

BRITO, J.; ALBUQUERQUE, E. M. Clusters industriais na economia brasileira: uma análise exploratória a partir de dados da RAIS. Estudos Econômicos, São Paulo, v. 32, n. 1, p. 71-102, 2002.

CARDOSO, Andreia S.C.S.C. Relatório de gestão e pesquisa de mercado do polo cerâmico de Eldorado dos Carajás. Belém: SEBRAE, 2013.

CASSIOLATO, J. E.; LASTRES, H. M. M. O foco em arranjos produtivos e inovativos locais de micro e pequenas empresas. [2003]. Disponível em: <http://www.ie.ufrj.br/redesist/P3/NTF2/Cassiolato\%20e\%20 Lastres.pdf >. Acesso em: 21 abr. 2014.

CASTRO, L. H. de. Arranjo produtivo local. Brasília: SEBRAE, 2009.

CROCCO, Marco Aurélio et al. Metodologia de identificação de arranjos produtivos locais potenciais: uma nota técnica. Belo Horizonte: UFMG, 2003.

CORDOVIL, G. V.; NAHUM, J. S. Indústrias cerâmicas e desenvolvimento territorial em São Miguel do Guamá, PA. Entre - Lugar, Dourados, ano 2, n. 4, p. 65-93, 2011.

DALLEMOLE, Dilamar; SANTANA, Antônio Cordeiro de. Concentração espacial e desenvolvimento local: cadeia produtiva de couro e derivados do Estado do Pará. Revista Desenvolvimento em Questão, ljuí, ano 6, n. 11, p. 99124, jan./jun. 2008. Disponível em: <http://www.redalyc.org/pdf/752/75211185004.pdf>. Acesso em: 21 jun. 2014.

ENRIQUEZ, M. Amélia R. da Silva. Mineração no Pará: uma agenda pró-ativa para ampliar e potencializar os benefícios regionais. Belém: FIEPA, 2007.

FÁVERO, L. P. L. et al. Análise de dados: modelagem multivariada para tomada de decisões. Rio de Janeiro: Elsevier, 2009.

INSTITUTO DO DESENVOLVIMENTO SOCIAL, ECONÔMICO E AMBIENTAL DO PARÁ (IDESP). Estatística Municipal.

Disponível em:<http://www.idesp.pa.gov.br/index.php/estatistica-municipal >. Acesso em: 20 abr. 2014. 
LOCKE, Richard M. Confiança e desenvolvimento local. Revista Econômica, Rio de Janeiro, v. 3, n. 2, p. 253281, dez. 2001.

MARSHALL, A. Princípios de economia. São Paulo: Abril Cultural, 1982.

MARTINS, Rafael D'Almeida; VAZ, José Carlos; CALDAS, Eduardo de Lima. A gestão do desenvolvimento local no Brasil: (des)articulação de atores, instrumentos e território. Revista de Administração Pública, Rio de Janeiro, v. 44, n. 3, p. 559-590, maio/jun. 2010.

PINHEIRO, A. M.; SOBREIRA, L. M. G.; RAPINI, M. Siqueira. Aglomerações produtivas no estado do Pará: uma proposta de análise para a construção civil. Revista Brasileira de Gestão e Desenvolvimento Regional, São Paulo, v. 4, n. 1, p. 24-56, jan./abr. 2008.

PORTER, M. E. Competição. Rio de Janeiro: Elsevier, 2009.

PUGA, Fernando Pimentel. Alternativas de apoio a MPMES localizadas em arranjos produtivos locais. Rio de Janeiro: BNDES, 2003.

SANTANA, A. Cordeiro de. Arranjos produtivos locais na Amazônia: metodologia para identificação e mapeamento. Belém: ADA, 2004.

SEBRAE. Termos de referência para atuação do Sistema SEBRAE em arranjos produtivos locais. Brasília: SEBRAE, 2003.

SCHIAVETTO, F.; ALVES, C. A. A Identificação dos Arranjos Produtivos Locais: uma Análise sobre sua Constituição no Contexto Regional e Nacional. Revista Eletrônica de Administração (REA), São Paulo, v. 13, n. 14, [não paginado], jan./jul. 2009.

SCHMITT, C.L; LOPES, H.C; D.; WITTMANN, M. L. Concentração de empresas: estratégia para a competitividade e a eficiência coletiva. In: Congresso Latino-Americano de Estratégias, 17., 2004, Itapema. Anais. Itapema: Univali, 2004. p. 1-15. 